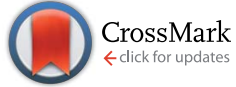

Cite this: RSC Adv., 2014, 4, 36117

Received 24th June 2014

Accepted 4th August 2014

DOI: $10.1039 / \mathrm{c} 4 \mathrm{ra06195k}$

www.rsc.org/advances

\title{
Dielectric properties of supramolecular ionic structures obtained from multifunctional carboxylic acids and amines $\uparrow$
}

\author{
Lidia González, Liyun Yu, Søren Hvilsted and Anne Ladegaard Skov*
}

\begin{abstract}
The dielectric properties of several supramolecular ionic polymers and networks, linked by the ammonium salts of hexamethylene diamine (HMDA), tris(2-aminoethyl)amine (TAEA), poly(propylene imine) (PPI) dendrimers and two short bis carboxymethyl ether-terminated poly(ethylene glycol)s (DiCOOH-PEG), are reported in this paper. All supramolecular ionic polymers and networks exhibit very high relative dielectric permittivities $\left(\varepsilon^{\prime}\right)\left(\sim 10^{2}-10^{6}\right)$ at low frequencies, and significantly lower values (from 1 up to 26) at high frequencies. Additionally, the dielectric properties of supramolecular ionic networks, formed by mixing multifunctional carboxylic acids such as citric acid (CA), tricarballylic acid (TCAA), trimesic acid (TMA), ethylenediaminetetraacetic acid (EDTA) and diethylenetriaminepentaacetic acid (DETPA) with two different Jeffamine polyetheramines (designated as D400 and D2000), are investigated. Here the relative dielectric permittivities of the supramolecular ionic structures formed with the multifunctional carboxylic acids were lower than those from the supramolecular ionic structures formed with the two carboxymethyl ether-terminated poly(ethylene glycol)s.
\end{abstract}

\section{Introduction}

A supramolecular structure arises as a result of reversible, noncovalent interactions between polymer chains or units on polymer chains. Examples of such non-covalent interactions are electrostatic ionic bonds, hydrophobic interactions, $\pi-\pi$ stacking or metal-ligand bonds and hydrogen bonds. ${ }^{1}$ Selfassembly, driven by hydrogen bonds with the participation of carboxylic groups, is a rich and highly important process in both nature and supramolecular chemistry. For instance, amino acids are a type of essential biomolecule endowed with carboxylic groups. The reversibility of these non-covalent interactions adds additional features to the material compared with common covalent polymers, thereby potentially leading to new properties such as improved processing and self-healing behaviour. Ionic self-assembly of telechelic polymers with ionic cross-linkers has been shown as an easy and versatile method for preparing supramolecular structures. ${ }^{2-5}$

High-permittivity materials are attractive for a broad range of applications where high electric energy storage is required. Examples of current materials with high relative dielectric permittivities are ferroelectric materials, ${ }^{6}$ which are used as capacitors in microelectronics. $\mathrm{BaTiO}_{3}, \mathrm{~Pb}(\mathrm{Zr}, \mathrm{Ti}) \mathrm{O}_{3}$ and $(\mathrm{Ba}, \mathrm{Sr})$

Danish Polymer Centre, Department of Chemical and Biochemical Engineering, Technical University of Denmark, Building 227, Søltofts Plads, DK-2800, Kgs. Lyngby, Denmark. E-mail: al@kt.dtu.dk; Fax: +45 4588 2258; Tel: +45 45252825

$\dagger$ Electronic supplementary information (ESI) available. See DOI: 10.1039/c4ra06195k
$\mathrm{TiO}_{3}$ (ref. 7 and 8) are examples of such materials and are widely used to manufacture electronic components. ${ }^{9}$

Another emerging area where dielectric permittivity is of great importance is in dielectric electroactive polymers, where the energy density of the materials scales with dielectric permittivity, and thus soft, high-permittivity elastomers are sought. ${ }^{10}$ The relative permittivities of conventional elastomers such as silicones, acrylics and polyurethanes are normally $\sim 3,5$ and 7 , respectively, at $1 \mathrm{~Hz}$. Composite materials can improve dielectric permittivity to a certain extent, but the addition of hard inorganic fillers to the soft elastomeric matrix introduces other unfavourable properties to the composite, such as increased Young's modulus, loss of tension and a significant Mullins effect. ${ }^{11}$ As an alternative the polymer chains in the elastomer are grafted to high-permittivity materials such as dipoles. Various methods have been applied in this respect, leading to high-permittivity elastomers with no increase in Young's modulus. ${ }^{\mathbf{1 2 - 1 4}}$ The approach can furthermore be combined with reducing the Young's modulus by creating bimodal networks. ${ }^{15-17}$ However, the method is still limited to an actuation improvement of a factor of the order of two.

As an alternative to the grafting of high-permittivity substituents, a family of polymeric supramolecular ionic structures, created from stoichiometric reactions between acid and base functional polymers, is investigated. The ionic crosslinker molecules are poly(propylene imine) (PPI) dendrimers, or multifunctional amines, which are, respectively, reacted with two carboxylic acid end-functionalised PEGs. The core molecules (PPI dendrimers or multifunctional amines) are then 


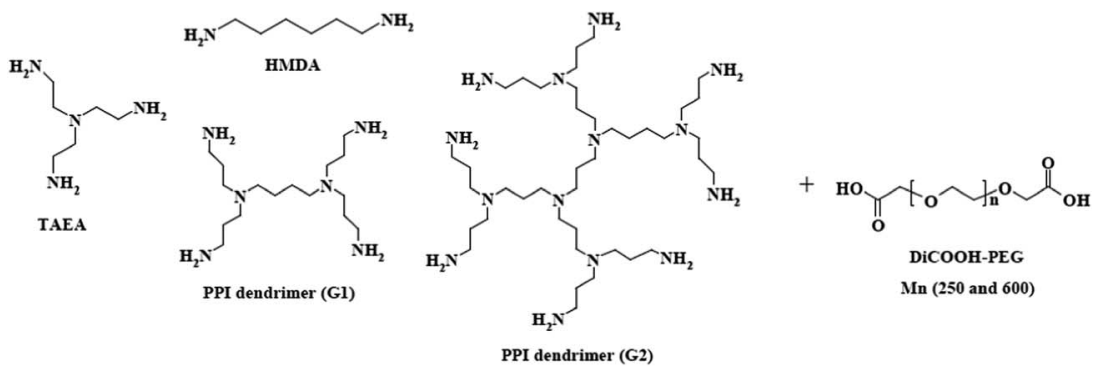

Fig. 1 Amines and carboxylic acid end-functionalised PEGs creating the supramolecular ionic structures.

linked ionically to carboxylic acids by corresponding ammonium salts. The focus in this study is on the dielectric properties of several supramolecular ionic structures derived from the protonation of hexamethylene diamine (HMDA), tris(2-aminoethyl)amine (TAEA) or dendritic amines (PPI G1 and PPI G2) with two carboxylic acid end-functionalised PEGs (DiCOOH-PEG 250 and 600, respectively) (Fig. 1). No data on dielectric properties of ionic networks has been published previously to our knowledge.

The reaction between a diamine such as HMDA and telechelic carboxylic acids causes the formation of - in ideal cases and with stoichiometric ratios - one long supramolecular polymer, while the reaction between multifunctional amines such as TAEA and PPI dendrimers and carboxylic acid end-functionalised PEGs (e.g. DiCOOH-PEGs) lead to supramolecular ionic networks. ${ }^{2}$ The proton transfer reaction from the carboxylic acid groups to the amino groups is a simple and fast way to prepare supramolecular ionic structures. These ionic structures, derived from ionic assembly between so-called Jeffamine polyetheramines and several multifunctional carboxylic acids such as citric acid (CA), tricarballylic acid (TCAA), trimesic acid (TMA), ethylenediaminetetraacetic acid (EDTA) and diethylenetriaminepentaacetic acid (DETPA) (Fig. 2), were investigated in relation to some of the multiple variables in a simple system. As illustrated in Fig. 2, Jeffamine D series products are amine-terminated PPGs.

\section{Experimental}

\section{Materials}

Poly(propylene imine) (PPI) dendrimers, based on the diaminobutane (DAB) core molecule of first- and second-generation
(PPI G1 and PPI G2), were purchased from SyMO-Chem BV (Eindhoven, The Netherlands) and used as received. Two bis carboxymethyl ether-terminated poly(ethylene glycol)s (designated as DiCOOH-PEG) were purchased from Aldrich and utilised as received $\left(M_{\mathrm{n}} \sim 250\right.$ and $\left.M_{\mathrm{n}} \sim 600\right)$. Hexamethylene diamine (HMDA), tris(2-aminoethyl)amine (TAEA), citric acid (CA), tricarballylic acid (TCAA), trimesic acid (TMA), ethylenediaminetetraacetic acid (EDTA) and diethylenetriaminepentaacetic acid (DETPA) were purchased from Aldrich and used as received, unless stated otherwise. Jeffamine polyetheramines (designated as D400 and D2000) were purchased from Huntsman, US, and used as received $\left(M_{\mathrm{n}}=430\right.$ and $\left.M_{\mathrm{n}}=2000\right)$.

\section{Preparation of the supramolecular ionic structures}

All supramolecular ionic structures are made from stoichiometric mixtures, if not stated otherwise. All of the supramolecular ionic structures described here are listed in Table 1. The ionic polymers and networks (see entries 1-12) were prepared simply by manually mixing stoichiometric amounts of HMDA, TAEA or poly(propylene imine) dendrimers (PPI G1 and PPI G2) and two different telechelic $(\alpha, \omega)$ dicarboxylic acids (DiCOOHPEG 250 and 600, respectively). No solvent was used. The network formation was directly observable, due to the exothermic nature of the reaction. Furthermore, we observed that while mixing the two components the viscosity of the mixtures increased. Moreover, a very detailed structural analysis of polymer and networks (entries $1-8$ ) by FTIR, ${ }^{1} \mathrm{H}$-NMR and ${ }^{13} \mathrm{C}$-NMR spectroscopies has been performed previously. ${ }^{2}$ The ionic networks (entries 13-19, Table 1) were prepared by manual mixing -followed by magnetically stirring stoichiometric amounts of

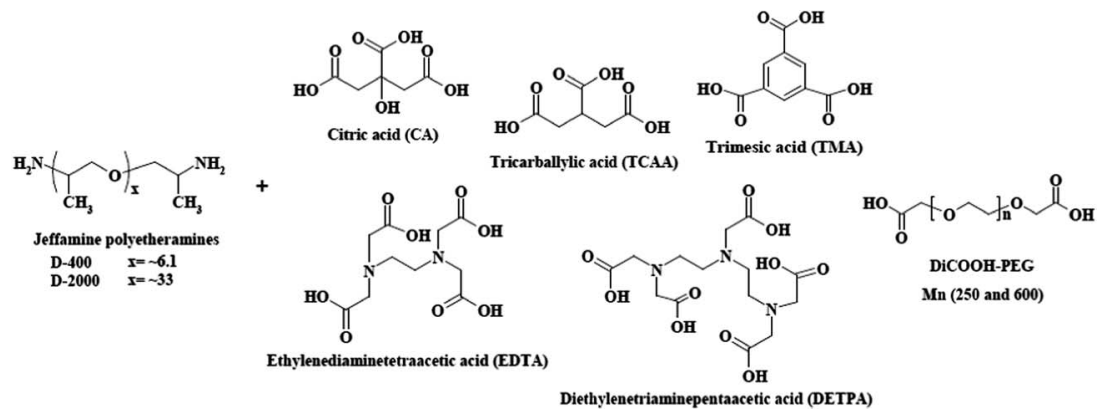

Fig. 2 Jeffamine polyetheramines (D400 and D2000) and several carboxylic acids used for the formation of supramolecular ionic polymers and networks. 
Table 1 Relative dielectric permittivities $\left(\varepsilon^{\prime}\right)$ of supramolecular ionic polymers and networks at $25^{\circ} \mathrm{C}$

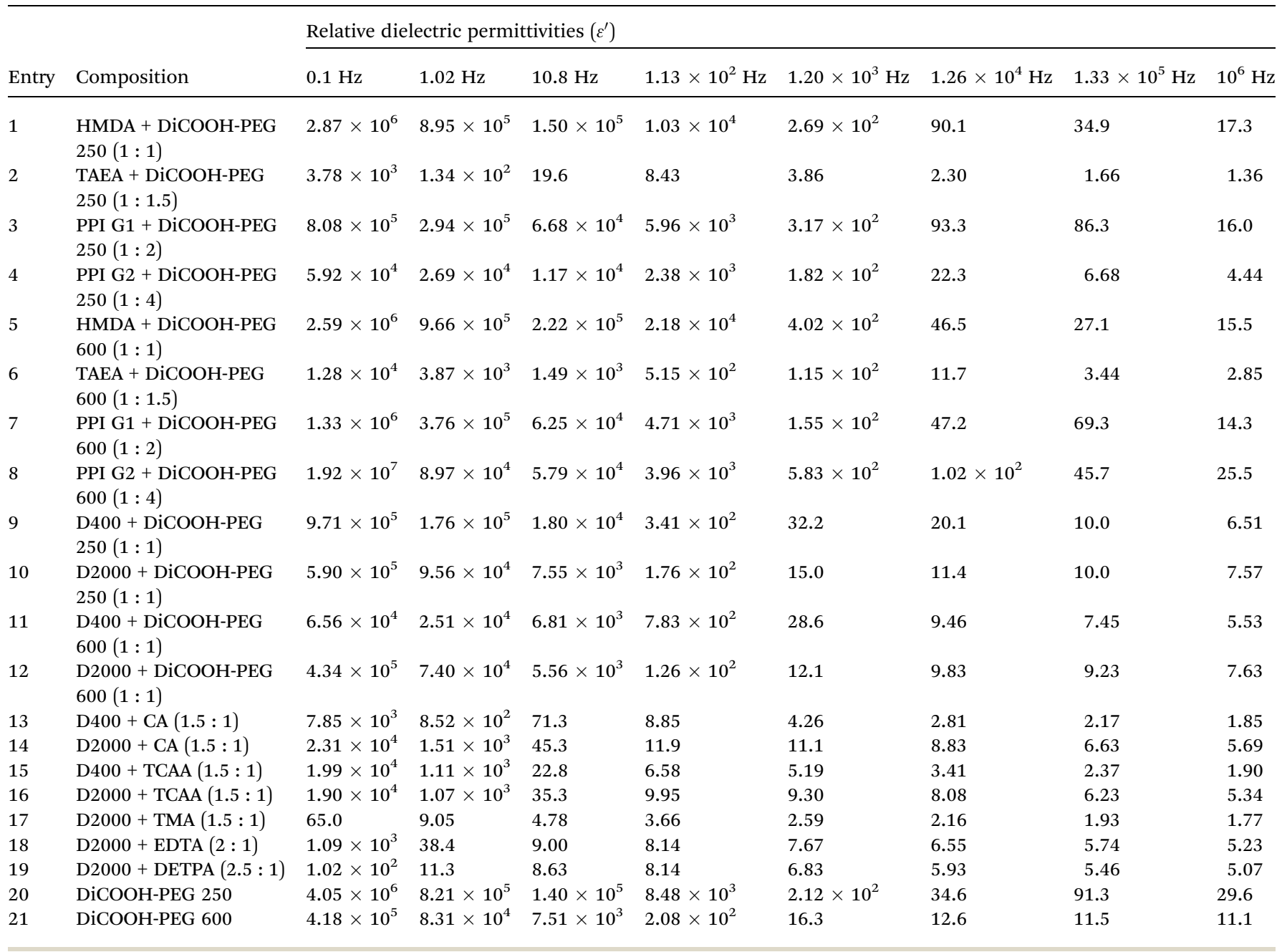

the different Jeffamine polyetheramines used (D400 and D2000) and several carboxylic acids (CA, TCAA, TMA, EDTA and DETPA), without the use of solvent and at different temperatures. The mixtures with CA and TCAA were heated for $30 \mathrm{~min}$ at $100{ }^{\circ} \mathrm{C}$, $30 \mathrm{~min}$ at $150{ }^{\circ} \mathrm{C}$ and finally $25 \mathrm{~min}$ at $200{ }^{\circ} \mathrm{C}$, since carboxylic acids (CA and TCAA) are solid at room temperature and have moderate-to-high melting points $\left(149-153{ }^{\circ} \mathrm{C}\right.$ and $156-161{ }^{\circ} \mathrm{C}$, respectively). The mixtures that contained TMA, EDTA and DETPA were heated for $60 \mathrm{~min}$ at $150{ }^{\circ} \mathrm{C}, 45 \mathrm{~min}$ at $200{ }^{\circ} \mathrm{C}$ and finally 5-7 min at $250^{\circ} \mathrm{C}$, since carboxylic acids (TMA, EDTA and DETPA) are solid at room temperature and have high melting points $\left(374-376{ }^{\circ} \mathrm{C}, 237-245^{\circ} \mathrm{C}\right.$ and $220-222^{\circ} \mathrm{C}$, respectively).

In order to avoid moisture uptake all samples were measured immediately after preparation.

\section{Characterization}

Water uptake of samples was determined from weighing samples before and after stay for 1 and 2 hours, respectively, in an air-conditioned chamber (Climacello Evo) at $25^{\circ} \mathrm{C}$ and $70 \%$ humidity. The glass transition temperatures for samples 10 and 12 were measured by employing a DSC Q1000 calorimeter
(TA Instruments) at heating/cooling rates of $10{ }^{\circ} \mathrm{C} \mathrm{min}^{-1}$. The samples weighed approximately $2-5 \mathrm{mg}$. The glass transition temperatures were calculated by means of a second heating scan based on the temperature of the halfway point of the jump in heat capacity when the material changed from the a glassy to a rubbery state. Thermal stability was assessed through thermogravimetry analysis employing a TGA Q500 instrument (TA Instruments) ranging from 20 up to $700{ }^{\circ} \mathrm{C}$ with a heating rate of $20^{\circ} \mathrm{C} \mathrm{min}-1$ under nitrogen flow.

Simple shear and oscillatory measurements were performed on an AR 2000 (TA Instruments). ETC standard aluminum parallel-plate geometry ( $25 \mathrm{~mm}$ diameter) was applied. Oscillatory experiments were performed in the linear viscoelastic (LVE) region in the frequency range of 0.01-100 Hz. Before applying frequency sweep steps, each sample was tested at the starting and final temperature to determine the range of its linear viscoelastic response (LVR). The tests were performed by strain sweeping at constant frequencies $(1$ and $100 \mathrm{~Hz}$ ), and the LVR range was determined as the region where the response of storage and loss moduli $\left(G^{\prime}\right.$ and $\left.G^{\prime \prime}\right)$ were constant over the entire range of strain for the two applied frequencies. After establishing the LVR region, a range of strains corresponding to 
the LVR was selected for sample evaluation, and the frequency sweeps were performed within a temperature range at about -20 to $120{ }^{\circ} \mathrm{C}$ depending on the investigated materials. When measurements were performed at temperatures ranging from 30 or $40{ }^{\circ} \mathrm{C}$ up to $120{ }^{\circ} \mathrm{C}$, the strain amplitude was kept at $2 \%$, which was ensured to be within the linear regime of all the materials. The strain amplitude was decreased slightly when measurements were performed at temperatures close to the glass transition temperature due to the resulting high torques.

Dielectric measurements were carried out by dielectric relaxation spectroscopy (DRS) with a Novocontrol Alpha-A highperformance frequency analyser. As the samples were liquidlike, a parallel plate liquid sample cell of $\varnothing=20 \mathrm{~mm}$ (BDS1308) was employed. The electrode gap was adjusted by silica spacers with a thickness of $50 \mu \mathrm{m}$.

\section{Results and discussion}

\section{General characterization}

The water uptake of two representative samples (samples 10 and 12) was evaluated at $25{ }^{\circ} \mathrm{C}$ and $70 \%$ humidity over 1 and 2 hours, respectively. D2000 + DiCOOH-PEG 250 (sample 10) had weight increases of $1.0 \%(1 \mathrm{~h})$ and $1.2 \%(2 \mathrm{~h})$ whereas D2000 + DiCOOH-PEG 600 (sample 12) showed a weight increase of 1.6\% $(1 \mathrm{~h})$ and $1.9 \%(2 \mathrm{~h})$. It was therefore concluded that the water uptake could be limited under normal laboratory conditions by measuring the mechanical and dielectric properties right after preparation of the ionic networks.

The determination of glass transition temperatures revealed that samples were homogeneous to a great extent since no significant changes were observed between the temperature cycles. If samples had been heterogeneous changing behavior upon the heating cycles would have been expected. Furthermore all samples were transparent with various yellow-red colors.

Rheological characterization showed that all samples obeyed time-temperature superposition, except the sample consisting of HMDA + DiCOOH-PEG 600 (sample number 5) for which a strong dependency on thermal history was observed which could indicate a heterogeneous structure due to the different relaxation phenomena going on in this supramolecular ionic polymer. As an example of a system showing very little or no dependency on thermal history, the LVE diagram for the ionic network of TAEA and DiCOOH-PEG is also shown in Fig. 3. Time-temperature superposed data for some representative

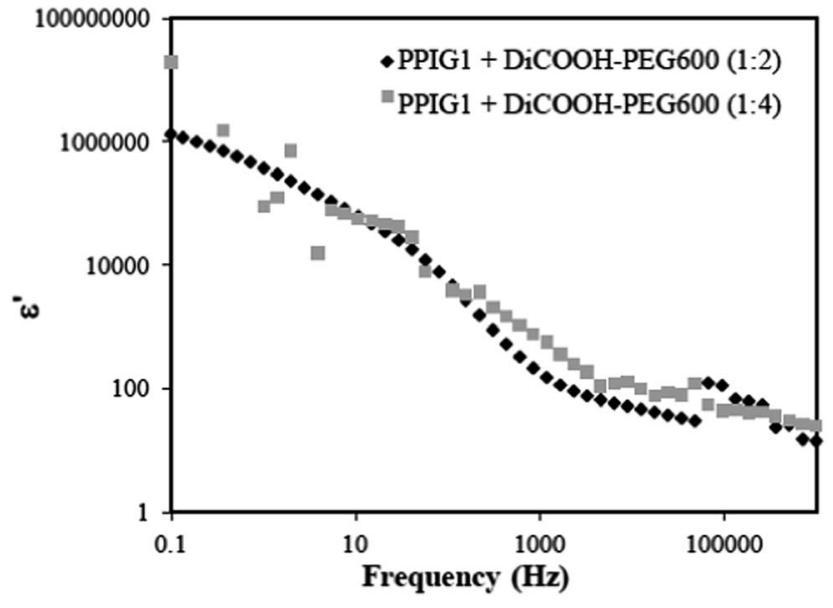

Fig. 4 Relative dielectric permittivities $\left(\varepsilon^{\prime}\right)$ of two ionic networks as a function of frequency at $25^{\circ} \mathrm{C}$.

samples can be seen in ESI, $\uparrow$ where the TTS technique is also explained further.

Furthermore from the rheological data it can be seen that all networks are liquid at $50{ }^{\circ} \mathrm{C}$ but their behavior at room temperature varies significantly depending on the reactants. Due to the polymeric origin of these ionic networks the elastic moduli are significantly smaller than that of the ionic networks of Aboudzadeh et al. ${ }^{4,5}$ and for further use as elastomer a reinforcement by e.g. fumed silica will be required. We have, however, decided, to focus on the unfilled elastomers in this work to look into which basis ionic networks provide the best dielectric properties.

\section{Dielectric properties}

The dielectric properties of the supramolecular ionic polymers and networks were investigated by dielectric relaxation spectroscopy (DRS). Fig. 4 shows the relative dielectric permittivities $\left(\varepsilon^{\prime}\right)$ of ionic networks, formed from the ammonium salts of poly(propylene imine) (PPI) dendrimers of first-(PPI G1) and second-(PPI G2) generation and bis carboxymethyl etherterminated poly(ethylene glycol)s (DiCOOH-PEG) $\left(M_{\mathrm{n}} \sim 600\right)$, as a function of frequency at $25^{\circ} \mathrm{C}$.

Both ionic networks possess similar dielectric properties. In addition, they showed very high relative dielectric permittivity $\left(\varepsilon^{\prime}\right)$ of the order of $10^{4}-10^{6}$ at low frequencies, with plateau-like
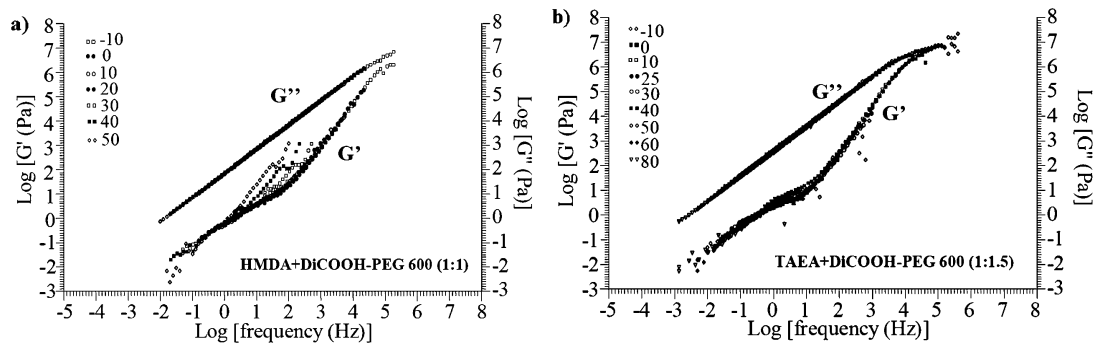

Fig. 3 Time-temperature superimposed rheological data for (a) the linear ionic assembly (HMDA + DiCOOH-PEG 600) and (b) the ionic network TAEA + DiCOOH-PEG 600. All data are superimposed to $50{ }^{\circ} \mathrm{C}$. 
behaviour at high frequencies and a relative dielectric permittivity of the order of 1-26, which resembles the dielectric permittivity of PEG. The fact that both ionic networks show high relative dielectric permittivities at low frequencies might be attributed to the existence of the so-called "space-charge or interfacial polarisation", ${ }^{18}$ which means that at low frequencies there is time for the charges to build up at the interface before the electric field changes direction, which in turn causes the high-permittivities. The space-charge polarisation mechanism takes place when charge carriers are present and can migrate an appreciable distance in a dielectric field. They are then trapped or cannot discharge at an electrode. This polarisation mechanism provides relative dielectric permittivities higher than orientation polarisation and is limited by voltage breakdown.

The dielectric properties of all supramolecular ionic structures studied here are summarised in Table 1. According to the results obtained, all supramolecular ionic structures had high relative dielectric permittivities $\left(\varepsilon^{\prime}\right)$ at low frequencies and low relative dielectric permittivities $\left(\varepsilon^{\prime}\right)$ at high frequencies with similar behaviours, as shown in Fig. 4. In general, the ionic structures formed between HMDA and the dicarboxylic acids PEGs (DiCOOH-PEG 250 and 600) (entry 1 and 5, Table 1) exhibited higher relative dielectric permittivities $\left(\varepsilon^{\prime}\right)$ compared to the ionic networks formed with TAEA, PPI G1 or PPI G2 and dicarboxylic acid PEGs (entry 2-4 and 6-8, Table 1). A possible explanation for this result could be that the dynamics of the supramolecular polymers formed from the two di-functional reactants (HMDA and DiCOOH-PEG 250/PEG 600) are much faster, since the resulting structure is not crosslinked and hence is a melt. In addition, it seems that ionic supramolecular structures formed with DiCOOH-PEG 600 (entry 5, Table 1) possess higher relative dielectric permittivities compared to ionic supramolecular structures formed with DiCOOH-PEG 250 (entry 1, Table 1$)$ at low frequencies $\left(0.1-1.20 \times 10^{3} \mathrm{~Hz}\right)$. This trend was observed for all pairs of supramolecular ionic polymers from DiCOOH-PEG 250 and 600 (entries 1-8, Table 1), which indicates that the low concentration of ionic groups facilitated interface polarisation. The opposite trend, as expected, was observed at high frequencies $\left(10^{4}-10^{6} \mathrm{~Hz}\right)$, since dielectric permittivity is expected to scale in line with the concentration of ionic groups.

From Table 1 it is also notable that the ionic network formed by mixing PPI G1 and DiCOOH-PEG 250 (entry 3, Table 1) showed higher $\varepsilon^{\prime}$ in the entire frequency range measured, compared to the network formed with PPI G2 and the DiCOOHPEG 250 (entry 4, Table 1). The number of primary amino groups located at the periphery of the PPI dendrimers, going from 4 in PPI G1 up to 8 in PPI G2, did not significantly influence the dielectric properties of the ionic networks. However, this may not be a general tendency, though for our particular systems the concentration of amines was similar for both dendrimers.

In order to compare dielectric properties as a function of cross-linking concentration $\left(\gamma_{\mathrm{c}}\right)$, crosslinking concentration has been calculated. Volume conservation is assumed since no significant volume changes could be observed upon mixing of the two reactants, in which case the concentration can then be calculated as:

$$
\gamma_{\mathrm{c}}=\frac{f_{1} \times\left(\frac{m_{1}}{M_{1}}\right)}{m_{1}+m_{2}}
$$

where $f_{1}$ is the functionality of molecule $1, m_{1}$ the mass of molecule $1, M_{1}$ the molecular weight of molecule 1 and $m_{2}$ is the mass of molecule 2. The results can be seen in Table 2. We have used the concentration based on mass rather than volume since there is little change in densities of the ionic supramolecular systems and for translating the concentrations into volume based one we should either rely on simplistic calculations of the densities or measure densities of all ionic structures.

Fig. 5 illustrates relative dielectric permittivities versus ionic cross-linking density for the ionic supramolecular structures

Table 2 lonic cross-linking densities $\left(\gamma_{c}\right)$ of supramolecular ionic polymers and network studies

\begin{tabular}{|c|c|c|c|c|c|c|}
\hline Entry & Composition & Molecule 1 & & Molecule 2 & & $\gamma_{\mathrm{c}}\left(\mathrm{mol} \mathrm{g}^{-1}\right)$ \\
\hline 1 & HMDA + DiCOOH-PEG 250 (1: 1) & $m_{1}=0.5$ & $f_{1}=2$ & $m_{2}=1.03$ & $f_{2}=2$ & 0.0056 \\
\hline 2 & TAEA + DiCOOH-PEG $250(1: 1.5)$ & $m_{1}=1.02$ & $f_{1}=3$ & $m_{2}=2.56$ & $f_{2}=2$ & 0.0074 \\
\hline 3 & PPI G1 + DiCOOH-PEG $250(1: 2)$ & $m_{1}=1$ & $f_{1}=4$ & $m_{2}=1.58$ & $f_{2}=2$ & 0.0049 \\
\hline 4 & PPI G2 + DiCOOH-PEG $250(1: 4)$ & $m_{1}=1$ & $f_{1}=8$ & $m_{2}=1.29$ & $f_{2}=2$ & 0.0045 \\
\hline 5 & HMDA + DiCOOH-PEG $600(1: 1)$ & $m_{1}=0.5$ & $f_{1}=2$ & $m_{2}=2.58$ & $f_{2}=2$ & 0.0028 \\
\hline 6 & TAEA + DiCOOH-PEG $600(1: 1.5)$ & $m_{1}=0.5$ & $f_{1}=3$ & $m_{2}=3.08$ & $f_{2}=2$ & 0.0036 \\
\hline 7 & PPI G1 + DiCOOH-PEG $600(1: 2)$ & $m_{1}=0.75$ & $f_{1}=4$ & $m_{2}=2.84$ & $f_{2}=2$ & 0.0026 \\
\hline 8 & PPI G2 + DiCOOH-PEG $600(1: 4)$ & $m_{1}=0.75$ & $f_{1}=8$ & $m_{2}=2.32$ & $f_{2}=2$ & 0.0025 \\
\hline 9 & D400 + DiCOOH-PEG $250(1: 1)$ & $m_{1}=3.02$ & $f_{1}=2$ & $m_{2}=1.76$ & $f_{2}=2$ & 0.0029 \\
\hline 10 & D2000 + DiCOOH-PEG $250(1: 1)$ & $m_{1}=4.01$ & $f_{1}=2$ & $m_{2}=0.5$ & $f_{2}=2$ & 0.00089 \\
\hline 11 & D400 + DiCOOH-PEG 600 (1:1) & $m_{1}=2.05$ & $f_{1}=2$ & $m_{2}=2.80$ & $f_{2}=2$ & 0.0020 \\
\hline 12 & D2000 + DiCOOH-PEG $600(1: 1)$ & $m_{1}=3.01$ & $f_{1}=2$ & $m_{2}=0.9$ & $f_{2}=2$ & 0.00077 \\
\hline 13 & $\mathrm{D} 400+\mathrm{CA}(1.5: 1)$ & $m_{1}=1$ & $f_{1}=3$ & $m_{2}=3.36$ & $f_{2}=2$ & 0.0036 \\
\hline 14 & $\mathrm{D} 2000$ + CA $(1.5: 1)$ & $m_{1}=0.21$ & $f_{1}=3$ & $m_{2}=3.12$ & $f_{2}=2$ & 0.00098 \\
\hline 15 & D400 + TCAA $(1.5: 1)$ & $m_{1}=1$ & $f_{1}=3$ & $m_{2}=3.66$ & $f_{2}=2$ & 0.0037 \\
\hline 16 & D2000 + TCAA $(1.5: 1)$ & $m_{1}=0.21$ & $f_{1}=3$ & $m_{2}=3.43$ & $f_{2}=2$ & 0.0010 \\
\hline 17 & D2000 + TMA (1.5: 1) & $m_{1}=0.30$ & $f_{1}=3$ & $m_{2}=4.29$ & $f_{2}=2$ & 0.00093 \\
\hline 18 & D2000 + EDTA (2:1) & $m_{1}=0.30$ & $f_{1}=4$ & $m_{2}=4.14$ & $f_{2}=2$ & 0.00092 \\
\hline 19 & D2000 + DETPA $(2.5: 1)$ & $m_{1}=0.21$ & $f_{1}=5$ & $m_{2}=2.54$ & $f_{2}=2$ & 0.00097 \\
\hline
\end{tabular}




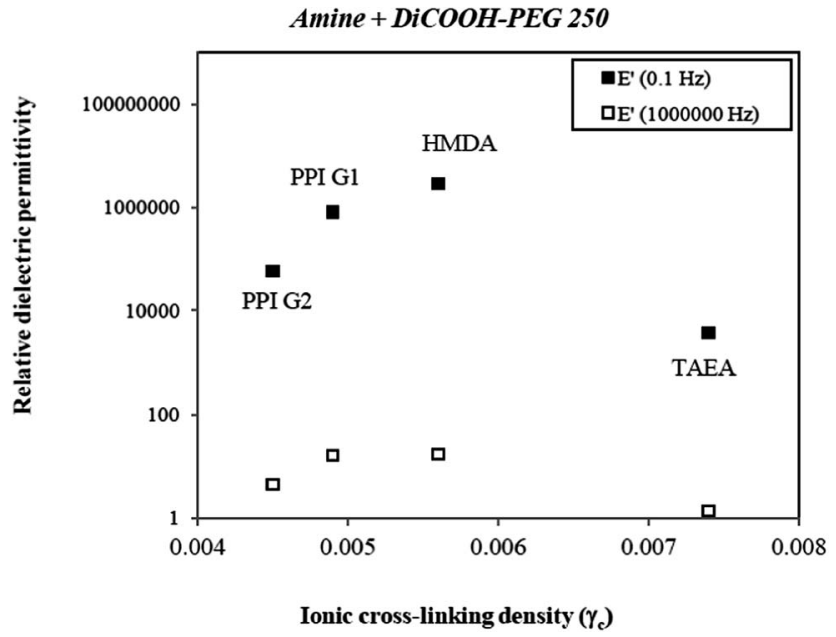

Fig. 5 Relative dielectric permittivities $\left(\varepsilon^{\prime}\right)$ at 0.1 and $10^{6} \mathrm{~Hz}$, respectively, of the ionic structures (four types of amines + DiCOOH-PEG 250) as a function of ionic cross-linking density.

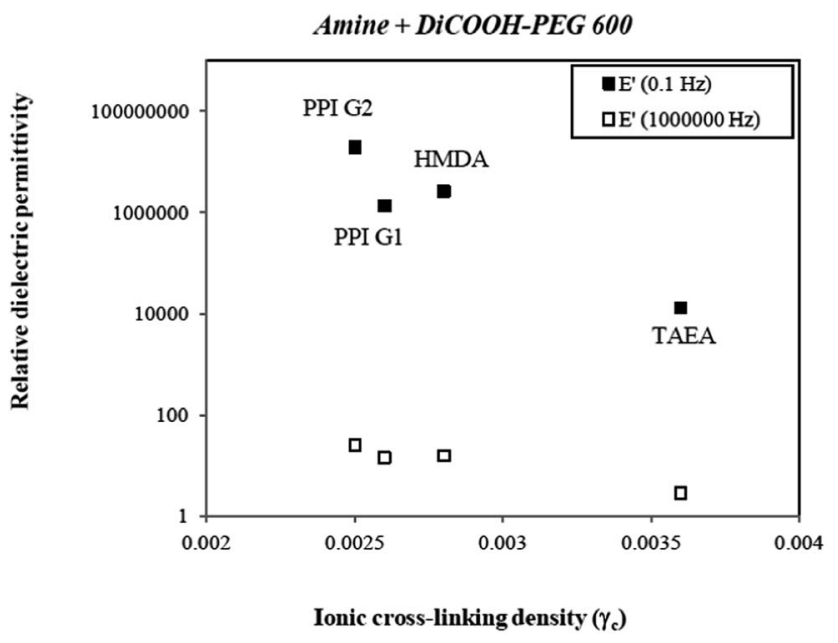

Fig. 6 Relative dielectric permittivities $\left(\varepsilon^{\prime}\right)$ at 0.1 and $10^{6} \mathrm{~Hz}$, respectively, of the ionic structures (four types of amines + DiCOOH-PEG $600)$ as a function of ionic cross-linking density. prepared by mixing different amines with DiCOOH-PEG 250 at 0.1 and $10^{6} \mathrm{~Hz}$, respectively. As can be seen, the ionic network TAEA + DiCOOH-PEG 250, which possesses the highest ionic cross-linking density, actually exhibits the lowest relative permittivities at both investigated frequencies. This may be due to the agglomeration of the ionic groups. Nevertheless, with the exception of this structure, the higher the ionic cross-linking density of the supramolecular ionic structures, the higher the relative dielectric permittivity.

Similar results were obtained when TAEA was mixed with a longer bis carboxymethyl ether-terminated poly(ethylene glycol) (DiCOOH-PEG 600) (see Fig. 6), where we noted that the higher the cross-linking density, the lower the relative permittivity. This might indicate that even though ionic networks formed with TAEA have the highest cross-linking density versus other corresponding supramolecular ionic structures, a number of other factors influence the permittivity of the materials. We also observe from Fig. 6 that the ionic network formed by mixing PPI G2 with DiCOOH-PEG 600, which has the lowest ionic crosslinking density, exhibits the highest relative permittivity at both given frequencies. The other supramolecular ionic structures showed the same trend.

From Table 1, we also observed that the supramolecular ionic polymers obtained from the Jeffamine polyetheramines (D400 and D2000) (entries 9-12, Table 1) exhibited lower relative dielectric permittivities in the entire measured frequency range, compared to the supramolecular ionic polymers obtained with HMDA. This result indicates that even though Jeffamine polyetheramines and HMDA have the same functionality $(f=2)$, the length of the amine - and therefore ionic cross-linking density has an effect on the dielectrical properties of the material (see also Fig. 7). We can also conclude that, in general, the longer the backbone of the Jeffamine polyetheramines used, the lower the relative dielectric permittivity. And finally, on comparison of the two supramolecular ionic polymers formed with different DiCOOH-PEGs (entries 9 and 11, Table 1), in general, the shorter the backbone of the DiCOOH-PEG, the higher the relative permittivity of the material.

The relative dielectric permittivities of the supramolecular ionic structures, formed with different multifunctional carboxylic acids (CA, TCCA, TMA, EDTA and DEPTA) and Jeffamine polyetheramines (D400 and D2000) (entries 13-19, Table 1),
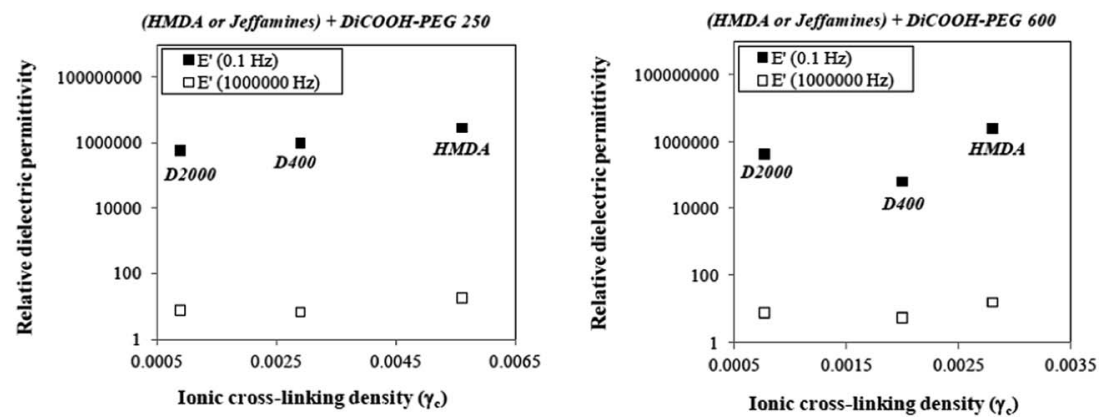

Fig. 7 Relative dielectric permittivities $\left(\varepsilon^{\prime}\right)$ at 0.1 and $10^{6} \mathrm{~Hz}$, respectively, of supramolecular ionic polymers from di-functional reactants as a function of ionic cross-linking density. 


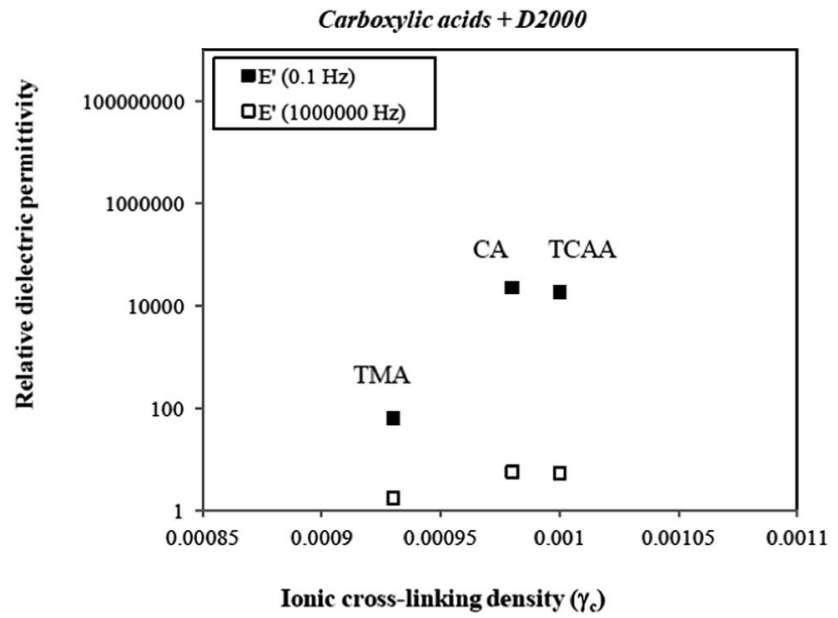

Fig. 8 Relative dielectric permittivities $\left(\varepsilon^{\prime}\right)$ at 0.1 and $10^{6} \mathrm{~Hz}$, respectively, of ionic structures as a function of ionic cross-linking density for various types of carboxylic acids.

were lower compared to the ionic structures mentioned above. Fig. 8 shows relative dielectric permittivities against ionic crosslinking density for the ionic structures prepared by mixing the carboxylic acids with a functionality of 3 (CA. TCAA or TMA) with the Jeffamine with the highest molecular weight (D2000) at 0.1 and $10^{6} \mathrm{~Hz}$, respectively.

As can be seen from Fig. 8, the supramolecular ionic structures prepared by using either citric acid (CA) or tricarballylic acid (TCAA) exhibited similar relative permittivity at the two measured frequencies. These results are in good agreement, since both molecules (CA and TCAA) have an almost identical

Table 3 Electrical conductivities $\left(\sigma^{\prime}\right)$ of the supramolecular ionic structures studied at $25^{\circ} \mathrm{C}$

\begin{tabular}{|c|c|c|c|}
\hline \multirow[b]{2}{*}{ Entry } & \multirow[b]{2}{*}{ Composition } & \multicolumn{2}{|c|}{$\begin{array}{l}\text { Electrical conductivities } \\
\left(\sigma^{\prime}\right)\left(\mathrm{S} \mathrm{m}^{-1}\right)\end{array}$} \\
\hline & & $10.8 \mathrm{~Hz}$ & $113 \mathrm{~Hz}$ \\
\hline 1 & HMDA + DiCOOH-PEG $250(1: 1)$ & $1.54 \times 10^{-4}$ & $2.77 \times 10^{-4}$ \\
\hline 2 & TAEA + DiCOOH-PEG $250(1: 1.5)$ & $1.67 \times 10^{-7}$ & $1.94 \times 10^{-7}$ \\
\hline 3 & PPI G1 + DiCOOH-PEG $250(1: 2)$ & $5.60 \times 10^{-5}$ & $1.12 \times 10^{-4}$ \\
\hline 4 & PPI G2 + DiCOOH-PEG $250(1: 4)$ & $5.75 \times 10^{-6}$ & $2.13 \times 10^{-5}$ \\
\hline 5 & HMDA + DiCOOH-PEG $600(1: 1)$ & $1.82 \times 10^{-4}$ & $3.92 \times 10^{-4}$ \\
\hline 6 & TAEA + DiCOOH-PEG $600(1: 1.5)$ & $8.26 \times 10^{-7}$ & $3.03 \times 10^{-6}$ \\
\hline 7 & PPI G1 + DiCOOH-PEG $600(1: 2)$ & $7.41 \times 10^{-5}$ & $1.26 \times 10^{-4}$ \\
\hline 8 & PPI G2 + DiCOOH-PEG $600(1: 4)$ & $4.41 \times 10^{-2}$ & $4.59 \times 10^{-2}$ \\
\hline 9 & D400 + DiCOOH-PEG $250(1: 1)$ & $3.28 \times 10^{-5}$ & $3.87 \times 10^{-5}$ \\
\hline 10 & D2000 + DiCOOH-PEG $250(1: 1)$ & $2.20 \times 10^{-5}$ & $2.46 \times 10^{-5}$ \\
\hline 11 & D400 + DiCOOH-PEG $600(1: 1)$ & $5.14 \times 10^{-6}$ & $1.24 \times 10^{-5}$ \\
\hline 12 & D2000 + DiCOOH-PEG $600(1: 1)$ & $1.76 \times 10^{-5}$ & $1.94 \times 10^{-5}$ \\
\hline 13 & $\mathrm{D} 400+\mathrm{CA}(1.5: 1)$ & $3.66 \times 10^{-7}$ & $4.01 \times 10^{-7}$ \\
\hline 14 & $\mathrm{D} 2000+\mathrm{CA}(1.5: 1)$ & $8.97 \times 10^{-7}$ & $9.09 \times 10^{-7}$ \\
\hline 15 & D400 + TCAA $(1.5: 1)$ & $7.37 \times 10^{-7}$ & $7.36 \times 10^{-7}$ \\
\hline 16 & D2000 + TCAA $(1.5: 1)$ & $7.75 \times 10^{-7}$ & $7.85 \times 10^{-7}$ \\
\hline 17 & D2000 + TMA $(1.5: 1)$ & $1.78 \times 10^{-8}$ & $2.25 \times 10^{-8}$ \\
\hline 18 & D2000 + EDTA $(2: 1)$ & $1.13 \times 10^{-7}$ & $1.13 \times 10^{-7}$ \\
\hline 19 & D2000 + DETPA $(2.5: 1)$ & $2.67 \times 10^{-8}$ & $3.04 \times 10^{-8}$ \\
\hline 20 & DiCOOH-PEG 250 & $1.57 \times 10^{-4}$ & $2.58 \times 10^{-4}$ \\
\hline 21 & DiCOOH-PEG 600 & $1.57 \times 10^{-5}$ & $1.91 \times 10^{-5}$ \\
\hline
\end{tabular}

chemical structure. This indicates that the hydroxyl group present in the TCAA structure does not influence the dielectrical properties of the material. Moreover, very low relative dielectric permittivities for the mixture prepared with the 3-foldsymmetric tricarboxylic acid (TMA) were obtained. This result can be attributed to a non-homogenous mixture, since mixing TMA into the Jeffamine was a rather difficult process because TMA has a high melting point $\left(374-376{ }^{\circ} \mathrm{C}\right)$. In addition, another hypothesis could be that the central planar orientation of the benzene cores of TMA has an impact on the organisation and self-assembly of the different molecules for the formation of a well-organised supramolecular ionic network.

The electrical conductivity of the high-permittivity materials is also of interest, since both dielectric electroactive polymers and capacitors in general need the lowest form of conductivity possible. Table 3 lists the electrical conductivities $\left(\sigma^{\prime}\right)$ of the supramolecular ionic structures studied in this work.

As can be seen from Table 3, all of the ionic structures exhibited low electrical conductivities (from $10^{-2}$ to $10^{-7} \mathrm{~S} \mathrm{~m}^{-1}$ ) at both of the frequencies evaluated in this paper. The ionic network that exhibited the highest electrical conductivity was prepared by mixing the PPI dendrimer generation 2 (PPI G2) with the dicarboxylic acid PEG (DiCOOH-PEG 600).

From Table 3 it is obvious that the materials are not ideal insulators as for example silicone, but on the other hand their conductivities remain lower than $\sim 10^{-4} \mathrm{~S} \mathrm{~m}^{-1}$, except for PPI G2 + DiCOOH-PEG 600 (1:4), which shows a slight leaning towards conducting behaviour.

\section{Conclusion}

The dielectric properties of a family of supramolecular ionic networks have been characterised by dielectric relaxation spectroscopy (DRS). All of the supramolecular ionic structures studied herein exhibited high relative dielectric permittivities $\left(\varepsilon^{\prime}\right)$ (from $10^{2}$ to $10^{6}$ ) at low frequencies, and lower values (from 1 up to 26) at high frequencies. The ionic networks that showed higher relative permittivities across the entire range of measured frequencies were formed by mixing generations 1 and 2 PPI dendrimers (PPI G1 and PPI G2) with the longest carboxylic acid end-functionalised PEG (DiCOOH-PEG 600). The supramolecular ionic polymer obtained by mixing HMDA and both carboxylic acid end-functionalised PEGs also exhibited high permittivity; however, it turned out that the ionic network obtained from trimesic acid (TMA) and Jeffamine D2000 showed very low levels of permittivity. In summary, the new high-permittivity supramolecular ionic structures described in this article are promising for the broad field of dielectric materials but much more data is required for obtaining structure-property relationships as it was shown that no clear relationship between the dielectric permittivity and the crosslinking concentration exists.

\section{Acknowledgements}

The authors gratefully acknowledge the financial support of InnovationsFonden. 


\section{References}

$1 \mathrm{~J}$. M. Lehn, Supramolecular Chemistry: Concepts and Perspectives, Wiley-VCH, Weinheim, Germany, 1995.

2 L. González, A. L. Skov and S. Hvilsted, J. Polym. Sci., Part A: Polym. Chem., 2013, 51(6), 1359-1371.

3 L. González, A. L. Skov and S. Hvilsted, Macromolecular Symposia - EUPOC, 2013, MS-Nr: masy.201300227.R2.

4 M. A. Aboudzadeh, M. E. Muñoz, A. Santamaría and D. Mecerreyes, RSC Adv., 2013, 3(23), 8677-8682.

5 M. A. Aboudzadeh, M. E. Muñoz, A. Santamaría, R. Marcilla and D. Mecerreyes, Macromol. Rapid Commun., 2012, 33(4), 314-318.

6 R. Ryoo, S. H. Joo, M. Kruk and M. Jaroniec, Adv. Mater., 2001, 13(9), 677-681.

7 J. Lee, S. Yoon, T. Hyeon, S. M. Oh and B. Kim, Chem. Commun., 1999, 21, 2177-2178.

8 S. Jun, S. H. Joo, R. Ryoo, M. Kruk, M. Jaroniec, Z. Liu, T. Ohsuna and O. Terasaki, J. Am. Chem. Soc., 2000, 122(43), 10712-10713.

9 W. Maison, R. Kleeberg, R. B. Heimann and S. Phanichphant, J. Eur. Ceram. Soc., 2003, 23(1), 127-132.
10 P. Brochu and Q. B. Pei, Macromol. Rapid Commun., 2010, 31(1), 10-36.

11 S. Vudayagiri, S. Zakaria, L. Y. Yu, S. S. Hassouneh, M. Benslimane and A. L. Skov, Smart Mater. Struct., accepted.

12 F. B. Madsen, A. E. Daugaard, S. Hvilsted, M. Y. Benslimane and A. L. Skov, Smart Mater. Struct., 2013, 22(10), 104002104012.

13 F. B. Madsen, I. Dimitrov, A. E. Daugaard, S. Hvilsted and A. L. Skov, Polym. Chem., 2013, 4(5), 1700-1707.

14 S. Risse, B. Kussmaul, H. Krüger and G. Kofod, RSC Adv., 2012, 2(24), 9029-9035.

15 F. B. Madsen, A. E. Daugaard, S. Hvilsted and A. L. Skov, RSC Adv., 2014, 4(14), 6939-6944.

16 A. G. Bejenariu, L. Y. Yu and A. L. Skov, Soft Matter, 2012, 8(14), 3917-3923.

17 A. L. Larsen, K. Hansen, P. Sommer-Larsen, O. Hassager, A. Bach, S. Ndoni and M. Jørgensen, Macromolecules, 2003, 36(26), 10063-10070.

18 J. R. MacCallum and C. A. Vincent, Polymer Electrolyte Reviews 2, Elsevier, London, 1989, pp. 43-58. 